\title{
Geometrical tests of cosmological models
}

\section{Calibration of rotational widths and disc scaling relations}

\author{
A. Saintonge ${ }^{1,2}$, K. L. Masters ${ }^{3}$, C. Marinoni ${ }^{4}$, K. Spekkens ${ }^{5,6}$, R. Giovanelli ${ }^{1}$, and M. P. Haynes ${ }^{1}$ \\ ${ }^{1}$ Department of Astronomy, Cornell University, Ithaca, NY 14853, USA \\ e-mail: amelie@physik.uzh.ch \\ 2 Institute for Theoretical Physics, University of Zurich, Winterthurerstrasse 190, 8057 Zurich, Switzerland \\ 3 Harvard-Smithsonian Center for Astrophysics, Cambridge, MA 02143, USA \\ ${ }^{4}$ Centre de Physique Théorique ${ }^{\star}$, CNRS-Université de Provence, Case 907, 13288 Marseille, France \\ 5 National Radio Astronomy Observatory (NRAO) ${ }^{\star \star}$ \\ 6 Department of Physics and Astronomy, Rutgers, the State University of New Jersey, Piscataway, NJ 08854, USA \\ e-mail: kmasters@cfa.harvard.edu; christian.marinoni@cpt.univ-mrs.fr; spekkens@physics.rutgers.edu; \\ [riccardo; haynes]@astro.cornell.edu
}

Received 17 January 2007 / Accepted 2 November 2007

\section{ABSTRACT}

\begin{abstract}
This is the second in a series of three papers dedicated to a new technique to select galaxies that can act as standard rods and standard candles in order to perform geometrical tests on large samples of high redshift galaxies to constrain different cosmological parameters. The goals of this paper are (1) to compare different rotation indicators in order to understand the relation between rotation velocities extracted from observations of the $\mathrm{H} \alpha \lambda 6563 \AA$ line and the [OII] $\lambda 3727 \AA$ line; and (2) to determine the scaling relations between physical size, surface brightness and magnitude of disc galaxies and their rotation velocity using the SFI++, a large catalog of nearby galaxies observed at $I$-band. A good correlation is observed between the rotation curve-derived velocities of the $\mathrm{H} \alpha$ and [OII] observations, as well as between those calculated from velocity histograms, justifying the direct comparison of velocities measured from $\mathrm{H} \alpha$ rotation curves in nearby galaxies and from [OII] line widths at higher redshifts. To provide calibration for the geometrical tests, we give expressions for the different scaling relations between properties of galaxies (size, surface brightness, magnitude) and their rotation speeds. We use Malmquist bias-corrected distances from the SFI++ template Tully-Fisher relation to generate a sizerotation velocity relation with unprecedentedly small scatter. We show how the best size-rotation velocity relation is derived when size is estimated not from disc scale lengths but from the isophotal diameter $r_{23.5}$, once these have been corrected for inclination and extinction effects.
\end{abstract}

Key words. galaxies: fundamental parameters - galaxies: evolution - cosmology: cosmological parameters cosmology: observations - galaxies: high-redshift

\section{Introduction}

With the new generation of telescopes and instruments, such as multi-object spectrographs and large field of view cameras, it has become possible to perform large, deep redshift surveys. Using these data, the structure and geometry of the Universe can be studied through new channels, namely geometrical tests such as the angular diameter test (angular size-redshift relation), the Hubble diagram (magnitude-redshift relation) and the Hubble test (count-redshift relation). These tests are generally difficult, requiring differentiation between the natural evolution of galaxies and the effects of geometry. Large redshift surveys ease the task in that respect.

To perform the geometrical tests mentioned above, a population of objects that can be tracked through redshift needs to be defined. More specifically, the angular diameter test requires a standard rod to be identified, the angular size of which is then

* Centre de Physique Théorique is UMR 6207 - "Unité Mixte de Recherche" of CNRS and of the Universities "de Provence", "de la Méditerranée" and "du Sud Toulon-Var" - Laboratory affiliated to FRUMAM (FR 2291).

$\star \star$ NRAO is a facility of the National Science Foundation, operated under cooperative agreement by Associated Universities, Inc. being measured over the redshift range of interest. The objects taken to serve that purpose could be anything from galaxies (e.g. Sandage 1972), clusters of galaxies (e.g. Sereno 2003; Cooray 1998), or dark matter halos (Cooray et al. 2001). Regardless of its nature, a standard rod should satisfy simple criteria: its size should be measured reliably up to high redshifts, and it should be observable in the local Universe to provide calibration. In this study, the relation between the physical size of a spiral galaxy and its rotation velocity (or global profile width) is used. By virtue of this relation, selecting a population of objects with a given rotation velocity is equivalent to selecting them based on their physical size.

In other papers of this series (Marinoni et al. 2008a,b, thereafter Paper I and Paper III), the angular diameter test is performed on a dataset of precursor Vimos/VLT Deep Survey (VVDS) (Le Fèvre et al. 2001) galaxies, using the linewidthdiameter relation to select standard rods. The test is used to isolate the effects of disk evolution with redshift, and to constrain the values of cosmological parameters. Though the VVDS provides excellent data at high redshift, the angular diameter test highlights one of its shortcomings: limited volume coverage at low redshift and therefore the lack of a comparison sample in the local Universe. 
In this context, the goal of this paper is twofold. The first one is to compare the rotation velocity indicator used for the high redshift data to those used in the local Universe, crosscalibrating rotation indicators used at different redshifts. This last point is of relevance since rotation information for spiral galaxies can be obtained through the observation of various spectral lines, the choice of which varies with the redshift of the sample. For galaxies with $z \lesssim 0.1$, the HI $21 \mathrm{~cm}$ line is an excellent candidate. The $\mathrm{H} \alpha \lambda 6563 \AA$ line is also frequently used for galaxies with low to moderate redshifts. However, the $\mathrm{H} \alpha$ line is quickly redshifted into the near-infrared and becomes unavailable to ground observers using optical telescopes for galaxies with $z \gtrsim 0.4$, even though with new near-infrared spectrographs such as SINFONI it is now possible to obtain $\mathrm{H} \alpha$ rotation curves for high redshift galaxies (e.g. Förster Schreiber et al. 2006). Even with the advent of such instruments, most large studies of galaxies at high redshift rely on the [OII] $\lambda 3727 \AA$ line, including the VVDS and the DEEP2 Redshift Survey (Davis et al. 2001). In order to compare sets of local and distant galaxies, it is therefore necessary to understand how rotation velocities extracted from these different lines relate. It has already been shown that velocity widths derived from $\mathrm{HI} 21 \mathrm{~cm}$ and $\mathrm{H} \alpha$ observations are in excellent agreement (see for example Courteau 1997; Vogt et al. 2004). The correlation between the [OII] and HI line widths has also been investigated (Kobulnicky \& Gebhardt 2000). Using a sample of 22 nearby late-type spirals they find [OII] widths to be accurate to within $10 \%$ for galaxies with a rotation width of $200 \mathrm{~km} \mathrm{~s}^{-1}$, which is comparable to the overall scatter in the local Tully-Fisher relation. However, they conclude that the uncertainties go up to about $50 \%$ for galaxies with widths $<150 \mathrm{~km} \mathrm{~s}^{-1}$. In this paper, a sample of 32 spiral galaxies with $0.155<z<0.25$ is used to compare velocity widths from the $\mathrm{H} \alpha \lambda 6563 \AA$ and [OII] $\lambda 3727 \AA$ lines (hereafter, $\mathrm{H} \alpha$ and [OII]). Even though these galaxies are more distant than in the Kobulnicky \& Gebhardt (2000) sample, the scatter in the rotation curves data points is significantly smaller, resulting in a better estimation of the rotation velocities. Our data also allows for a direct comparison between $\mathrm{H} \alpha$ and [OII], therefore bridging nicely the gap between low and high redshift samples. Contrary to the Kobulnicky \& Gebhardt (2000) study, the galaxy sample used here is restricted to late spiral types, therefore we will not address the question of possible biasses due to varying morphologies.

The second goal of this paper is to establish, using a large sample of nearby galaxies (SFI++; Springob et al. 2007, hereafter S07), the physical properties of the standard rods and standard candles used to perform the geometrical tests, free from any evolutionary biases. This is especially useful for calibrating the angular diameter relation and for separating the effects of galaxy evolution from those of geometrical variations (see Paper I). By establishing clearly the scaling relations between the size, surface brightness and magnitude of these nearby galaxies observed at $I$-band and their rotation velocity, we establish the calibration that will allow us to select standard rods/candles simply by tracing through redshift galaxies with a given rotation speed. Comparisons between these scaling relations and theoretical predictions also provide important constraints on galaxy formation scenarios within the canonical cosmological framework (e.g. Dalcanton et al. 1997; Mo et al. 1998; Dutton et al. 2007) and provide an important low-redshift benchmark for evolutionary studies of these properties (e.g. Somerville et al. 2006).

In Sect. 2 a description of the galaxies observed and of the data reduction process is given, while in Sect. 3 we describe how velocity widths were extracted from the data and how they compare for both sets of emission lines. In Sect. 4 we present a sample of nearby galaxies used as calibrators for the purpose of the angular diameter test (Paper III) and discussion of our results are given in Sect. 5. Calculations were done assuming $H_{0}=70 \mathrm{~km} \mathrm{~s}^{-1} \mathrm{Mpc}^{-1}, \Omega_{\mathrm{M}}=0.3, \Omega_{\Lambda}=0.7$.

\section{Data}

\subsection{Target selection and observational setup}

Using the Hale $5 \mathrm{~m}$ telescope of Mount Palomar during the course of three short observing runs between 2003 March and 2004 February (total of 12 nights), long-slit spectra were obtained for a sample of spiral galaxies. The galaxies were chosen in the area covered by the Data Release 2 of the Sloan Digital Sky Survey ${ }^{1}$ (SDSS, Stoughton et al. 2002), and selected to have inclinations in excess of 50 degrees, Petrosian radii containing $90 \%$ of the light in $r$ band larger than $4^{\prime \prime}$ to avoid AGNdominated systems, and [OII] $\lambda 3727 \AA$ equivalent widths in the range of 10-35 $\AA$. The galaxies were also required to be in the redshift range $0.155<z<0.25$. Galaxies with close neighbours or disturbed morphologies were rejected to avoid including interacting systems in the sample.

Using the Double Spectrograph (Oke \& Gunn 1982), both $\mathrm{H} \alpha$ and [OII] emission lines could be observed simultaneously. The main properties of the galaxies are listed in Table 1. The first two columns are the AGC (Arecibo General Catalog) and SDSS names, respectively (the J2000 coordinates of the objects can be directly extracted from their SDSS name). The third column is the heliocentric systemic velocity of the galaxies, as measured from the $\mathrm{H} \alpha$ line. All other parameters (redshift, I-band magnitude, disc scale-length, inclination and position angle measured counterclockwise from North) are from the SDSS database. Unfortunately, about $50 \%$ of the observing time was lost to bad weather conditions, forcing the final sample presented in this paper to contain only the 32 galaxies for which it was possible to get rotation curves in both $\mathrm{H} \alpha$ and [OII] .

The red camera of the Double Spectrograph was used with a 1200 lines $\mathrm{mm}^{-1}$ grating (7100 $\AA$ blaze) set at an angle of $47^{\circ} 18^{\prime}$ in order to cover the wavelength range of 7520-8185 $\AA$. The spectral resolution is $0.65 \AA$ pixel $^{-1}$ with $24 \mu \mathrm{m}$ pixels and the spatial scale is of 0.468 pixel $^{-1}$. The CCD camera had a size of $1024 \times 1024$ pixels, and a $1^{\prime \prime} \times 128^{\prime \prime}$ slit was used (except for a few galaxies where the $2^{\prime \prime} \times 128^{\prime \prime}$ slit was used due to very poor seeing conditions). The blue camera was set to cover the range 3675-5140 A by using a 1200 lines $\mathrm{mm}^{-1}$ grating with a $4700 \AA$ blaze at an angle of $35^{\circ} 52^{\prime}$. Using the $2788 \times 512$ chip $(15 \mu \mathrm{m}$ pixels), a spatial sampling of 0.390 pixel $^{-1}$ was achieved, as well as a $0.55 \AA$ pixel $^{-1}$ spectral resolution.

\footnotetext{
${ }^{1}$ Funding for the creation and distribution of the SDSS Archive has been provided by the Alfred P. Sloan Foundation, the Participating Institutions, the National Aeronautics and Space Administration, the National Science Foundation, the US Department of Energy, the Japanese Monbukagakusho, and the Max Planck Society. The SDSS Web site is http://www.sdss.org/. The SDSS is managed by the Astrophysical Research Consortium (ARC) for the Participating Institutions. The Participating Institutions are The University of Chicago, Fermilab, the Institute for Advanced Study, the Japan Participation Group, The Johns Hopkins University, Los Alamos National Laboratory, the Max-Planck-Institute for Astronomy (MPIA), the Max-Planck-Institute for Astrophysics (MPA), New Mexico State University, University of Pittsburgh, Princeton University, the United States Naval Observatory, and the University of Washington.
} 
Table 1. Properties of the galaxy sample.

\begin{tabular}{|c|c|c|c|c|c|c|c|}
\hline AGC \# & Name & $\begin{array}{c}v_{0} \\
\mathrm{~km} \mathrm{~s}^{-1}\end{array}$ & $z_{\text {SDSS }}$ & $\begin{array}{c}m_{i} \\
\mathrm{mag}\end{array}$ & $\begin{array}{l}r_{\mathrm{D}} \\
\mathrm{kpc}\end{array}$ & $\begin{array}{c}i \\
\operatorname{deg}\end{array}$ & $\begin{array}{l}\text { PA } \\
\text { deg }\end{array}$ \\
\hline 101738 & SDSS J001107.7+003551.7 & 46600 & 0.155 & 17.81 & 4.24 & 66 & 8 \\
\hline 101701 & SDSS J003123.8+141313.8 & 49444 & 0.165 & 17.15 & 6.93 & 67 & 116 \\
\hline 112393 & SDSS J014451.5+133616.9 & 46711 & 0.156 & 17.31 & 6.18 & 54 & 111 \\
\hline 130796 & SDSS J031818.8+010750.4 & 48640 & 0.162 & 17.68 & 3.29 & 51 & 87 \\
\hline 431415 & SDSS J032434.8-071050.6 & 49673 & 0.166 & 17.32 & 7.82 & 64 & 356 \\
\hline 130826 & SDSS J033331.8+010717.1 & 53337 & 0.178 & 17.48 & 6.92 & 61 & 158 \\
\hline 431418 & SDSS J040000.2-053852.3 & 50138 & 0.167 & 17.36 & 7.03 & 66 & 51 \\
\hline 171385 & SDSS J073235.0+374734.4 & 65413 & 0.219 & 16.94 & 9.09 & 62 & 331 \\
\hline 171389 & SDSS J074608.6+395658.5 & 47142 & 0.157 & 17.46 & 5.54 & 49 & 343 \\
\hline 171394 & SDSS J075555.0+364057.7 & 55351 & 0.185 & 17.01 & 7.60 & 49 & 103 \\
\hline 181489 & $\mathrm{~J} 080438.6+46$ & 60671 & 0.202 & 17.12 & 6.00 & 55 & 12 \\
\hline 181487 & $\mathrm{~J} 081345.7+4$ & 54478 & 0.182 & 17.40 & 6.28 & 61 & 322 \\
\hline 181490 & SDSS J081750.4+4 & 55617 & 0.186 & 17.30 & 7.82 & 56 & 35 \\
\hline 181488 & J084304.2- & 49300 & 0.165 & 17.40 & 8.79 & 63 & 7 \\
\hline 191728 & J091957.0+013 & 52850 & 0.176 & 16.90 & 5.51 & 51 & 20 \\
\hline 191729 & SDSS J092050.9+575758.5 & 51558 & 0.172 & 16.94 & 6.01 & 46 & 117 \\
\hline 191731 & SDSS J094412.5+020206.5 & 47453 & 0.158 & 17.17 & 6.30 & 60 & 99 \\
\hline 191732 & SDSS J100047.6+020406.6 & 55778 & 0.185 & 16.71 & 5.02 & 61 & 90 \\
\hline 501752 & SDSS J100757.3-005612.3 & 46402 & 0.155 & 17.08 & 4.38 & 58 & 179 \\
\hline 202041 & SDSS J104215.5+010508.3 & 58452 & 0.195 & 17.46 & 7.47 & 60 & 104 \\
\hline 501753 & SDSS J110117.7-010014.9 & 47277 & 0.158 & 17.39 & 7.15 & 66 & 36 \\
\hline 212861 & SDSS J110936.9+010957.8 & 63918 & 0.213 & 17.42 & 9.08 & 60 & 95 \\
\hline 511373 & SDSS J114001.2-004624.3 & 56368 & 0.188 & 17.36 & 5.25 & 58 & 107 \\
\hline 212862 & $14919.0+001806.3$ & 48216 & 0.161 & 17.14 & 6.40 & 53 & 102 \\
\hline 511374 & SDSS J115640.0-005128.6 & 50585 & 0.169 & 17.13 & 6.66 & 55 & 122 \\
\hline & & & 0.1 & 17.03 & 6.06 & 54 & 41 \\
\hline 224090 & SDSS J124819.3+672312.2 & 50790 & 0.170 & 17.38 & 7.56 & 51 & 26 \\
\hline 224092 & SDSS J125522.8+672306.8 & 49401 & 0.165 & 17.51 & 6.73 & 55 & 173 \\
\hline 242064 & SDSS J141054.1+013714.3 & 51706 & 0.1 & 17.32 & 8.46 & 49 & 129 \\
\hline 242065 & SDSS J141322.7+644404.7 & 54763 & 0.183 & 17.51 & 5.04 & 53 & 79 \\
\hline 242067 & SDSS J142846.2-000245.6 & 55232 & 0.184 & 17.23 & 7.75 & 58 & 242 \\
\hline 332257 & SDSS J234325.9+005605.2 & 67105 & 0.224 & 18.20 & 7.45 & 64 & 51 \\
\hline
\end{tabular}

The exposure time was of $3600 \mathrm{~s}$ for most galaxies, though it was extended to $4800 \mathrm{~s}$ for a few sources when the flux in the [OII] line was very low. Sky transparency and seeing conditions varied greatly over the course of the observing runs, but most observations were made between $1^{\prime \prime}$ and $2^{\prime \prime}$ seeing. In order to align the slit with the major axis of the galaxies, the ring angle of the spectrograph was set using the position angles of the galaxies given in the SDSS database. For each galaxy, the ring angle used is given in Table 1. On each night of observing, standard calibration images were obtained: dome flats, twilight flats (for the blue camera only), bias frames and darks. Given observing conditions, no attempt was made for any photometric calibration.

\subsection{Data reduction}

The data from both cameras were reduced independently using tasks from the Image Reduction and Analysis Facility (IRAF) (Tody 1993). Images from the blue camera had to be reduced with particular caution due to the presence of many bad columns and other defects in the CCD. First, all the images were trimmed to remove overscans and low sensitivity regions on the edges. Then flat fields were constructed separately for each camera and for each night using the dome flats, as well as the twilight flats in the case of the blue images. After this, the images were also corrected for bad pixels using a mask created from twilight flats and the effects of cosmic rays removed.

The cleaned images were wavelength calibrated and corrected for s-shape distortion. This was done in the dispersion direction by using observations of a reference star that was positioned at five different places along the slit, and for the spatial direction by using either strong sky lines (red camera images) or lamp spectra (blue camera images). The correction was in some cases important, especially in the dispersion direction where it was generally on the order of a few pixels. Individual exposures of each galaxy were then appropriately shifted and stacked. For these combined images, the background was fitted in order to remove the sky lines. The process allowed for optimal removal of the sky lines falling near or on the galaxy emission lines of interest. The continuum was finally subtracted from these background corrected frames.

\section{Comparing and calibrating rotation velocity indicators}

Here we describe the techniques we use to extract rotation velcoity information from the reduced and calibrated spectra (see the previous section), and compare results from different methods. We were able to extract rotation velocity information for galaxies in our Palomar sample (hereafter refered to as the $z 0.2$ sample) from both $\mathrm{H} \alpha$ and [OII] emission lines, using both rotation curves and velocity histograms.

\subsection{Rotation curve extraction and modeling}

A Gaussian was fitted along the dispersion axis at every pixel inside a box encompassing the emission line (either $\mathrm{H} \alpha$ or $[\mathrm{OII}]$ ). In the case of the [OII] line where the signal-to-noise ratio $(S / N)$ was often smaller than 3 , Hanning smoothing was performed 
before the Gaussian fitting took place. Then, the rotation curves were calibrated, the measured wavelength converted to a velocity and the radial axis scaled to arcseconds. Following this, each point of the fit was examined and either rejected or included in the rotation curve. The criteria were a minimal $S / N$ for the fitted Gaussian (4 for $\mathrm{H} \alpha$ and 3 for [OII]) and a maximum width of $400 \mathrm{~km} \mathrm{~s}^{-1}$. The resulting rotation curves were then examined and some rejected points reintroduced, while some others that satisfied the criteria were judged unrealistic and manually removed. These points for which a manual intervention was required were almost all either in the outer regions of the galaxy where the $S / N$ drops significantly or contaminated by sky lines or cosmic rays that were not completely successfully removed. Finally, the center of light of the galaxy was determined and used to center the rotation curve along the spatial direction. This procedure is similar to the ones presented in Vogt et al. (2004) and Catinella et al. (2005).

The rotation curves were subsequently modeled by first folding about the kinematic center and fitting a Polyex model (Giovanelli \& Haynes 2002) of the form:

$V(r)=a_{1}\left(1-\mathrm{e}^{-a_{2} r}\right)\left(1+a_{3} r\right)$.

The parameter $a_{1}$ is related to the maximal velocity of the rotation curve and $a_{2}$ controls the exponential rise of the rotation curve and the position of the "elbow", the point where a transition occurs between the steeply rising inner part and the flat outer region. Finally, $a_{3}$ controls the slope of the outer part of the rotation curve. Due to the small radial extent of the emission lines, the value of $a_{3}$ is meaningless in most cases and has been manually set to zero. We also modeled our data with the universal rotation curves of Persic et al. (1996), but found the Polyex model to deliver greater flexibility, as in Catinella et al. (2007). The $\mathrm{H} \alpha$ rotation curves are shown for all galaxies in Fig. 1, and the [OII] rotation curves in Fig. 2. The solid lines are the Polyex fits, while the vertical dotted lines show the position of $r_{83}$, the radius containing $83 \%$ of the light of the galaxy. There are no steeply rising rotation curves in our sample because of beam smearing, the well-known tendency of seeing effects to bias velocities in the inner parts of the rotation curves low (e.g. van den Bosch et al. 2000; Swaters et al. 2000; Weiner et al. 2006). The velocity widths that are derived in Sect. 3.2 are however not affected by this effect, since the rotation curves mostly extend to radii where they reach their plateau.

In Fig. 3, the Polyex fits to both the $\mathrm{H} \alpha$ and the [OII] rotations curves are shown together when sufficient data was available in both spectral lines to allow for the fitting process. In all the cases where the [OII] line was bright enough for the rotation curve to be traced out to the region where it starts flattening out, the agreement between the two sets of rotation curves is excellent. In the other cases, the trend is for the [OII] rotation curves to be slightly shallower.

Using these fits, the rotational velocity of each galaxy was determined, both for its $\mathrm{H} \alpha$ and [OII] lines. The velocity adopted for a given rotation curve is the value of the Polyex fit at a radius corresponding to $r_{83}$. The values for $r_{83}$ are taken to be $3.5 \times$ $r_{\mathrm{D}}$, where $r_{\mathrm{D}}$ is the disk scale length. Since all the galaxies in the sample were taken from the SDSS, the values of physical parameters were taken from their database. The scale lengths are from an exponential fit performed on the $i$-band image of each galaxy. These values are presented in Col. 5 of Table 1 . The value of $r_{83}$ for each galaxy is also represented in Figs. 1 and 2 by the vertical dotted lines. Also, Tables 2 and 3 present the values of the Polyex coefficients and the rotation velocity at $r_{83}, v_{\text {rot }}\left(r_{83}\right)$, for the $\mathrm{H} \alpha$ and $[\mathrm{OII}]$ data, respectively.
In Fig. 4, we compare the rotation velocities that come from the $\mathrm{H} \alpha$ and [OII] rotation curves. Following the prescription of Isobe et al. (1990), a best fit to the data was obtained by linear regression using an ordinary least-squares bisector method (also called "double regression"). The two outlying data points were ignored during the fitting. The solid line in Fig. 4 is the best-fit relation and the two dashed lines are the minimum and maximum slopes allowed by the best-fit parameters. The dotted line is the 1:1 relation. The standard deviation of the residuals of the best-fit (shown in the bottom panel) is $28.6 \mathrm{~km} \mathrm{~s}^{-1}$.

\subsection{Velocity histograms}

In order to cross-correlate the results with techniques used at higher redshift, measurements of the rotation velocities using velocity histograms were also made. These velocity histograms were built by collapsing the two-dimensional images along the spatial direction in order to form one-dimensional spectra. To determine the region of the image that will be collapsed, the central position of the continuum along the spatial direction as well as its width were determined by fitting a simple Gaussian. This region needs to be large enough to include all the emission from the galaxy, but not unnecessarily wide to avoid increasing the noise in the one-dimensional spectra. Since some of the galaxies have their emission lines extending slightly beyond the continuum, everything within $5 \sigma$ of the center of the continuum was selected and added together, thus producing an intensity weighted spectrum.

A Gaussian was fitted to each of the $\mathrm{H} \alpha$ histograms. The full width at half maximum ( $F W H M$ ) of that Gaussian was converted into an estimate of the rotation velocity of the galaxy, $\sigma_{\text {rot }}$, via:

$$
\sigma_{\text {rot }}=\frac{\left(F W H M_{l}^{2}-F W H M_{i}^{2}\right)^{1 / 2}}{2.355} \cdot \frac{c}{\lambda_{0}(1+z)} \cdot \frac{1}{\sin (i)}
$$

where $F W H M_{l}$ is the $F W H M$ of the emission line histogram, $F W H M_{i}$ the instrumental broadening, $\lambda_{0}$ the rest-frame wavelength of the emission line and $i$ the inclination of the disk. The inclinations were determined from the axial ratio of the disks as derived from the same SDSS fits that produced the disk scale lengths. In the case of the $\mathrm{H} \alpha$ lines, $F W H M_{i}$ was obtained by fitting a Gaussian to a bright night sky line that falls near the emission line. The velocities obtained using this technique $\left(\sigma_{\text {rot }}\right)$ are plotted against the velocities derived from the rotation curves $\left(v_{\text {rot }}\right)$ for the $\mathrm{H} \alpha$ emission on the top panel of Fig. 5 and are presented in Col. 6 of Table 2. In Fig. 5 we plot only the galaxies for which the rotation curve extends radially far enough to reliably determine a rotation velocity. The standard deviation of the residuals of the $\sigma_{\text {rot }}(\mathrm{H} \alpha)-v_{\text {rot }}(\mathrm{H} \alpha)$ from the 1:1 relation is $20.8 \mathrm{~km} \mathrm{~s}^{-1}$, which represents an error of $\sim 10 \%$ for a galaxy with a rotation velocity of $200 \mathrm{~km} \mathrm{~s}^{-1}$.

For the [OII] emission, one-dimensional spectra were produced in the same manner, but this time $\sigma_{\text {rot }}$ cannot be calculated by a single Gaussian fit since the doublet nature of the line needs to be taken into account. Instead, deblending of the two lines was done using the "splot" procedure in IRAF. The expected positions of the two lines of the doublet were given as input, and the relative position of the two lines is maintained fixed during the fitting process. We farther required that the width of the two lines be the same (as it should be for physical reasons), and that the amplitude of the two lines be in a reasonable ratio (not more than a factor of three between the two, which is similar to the criteria of Kobulnicky \& Gebhardt 2000). Since the process relies significantly on subjective judgement on the part of the data processor, especially with regard to the size of the fitting region around 


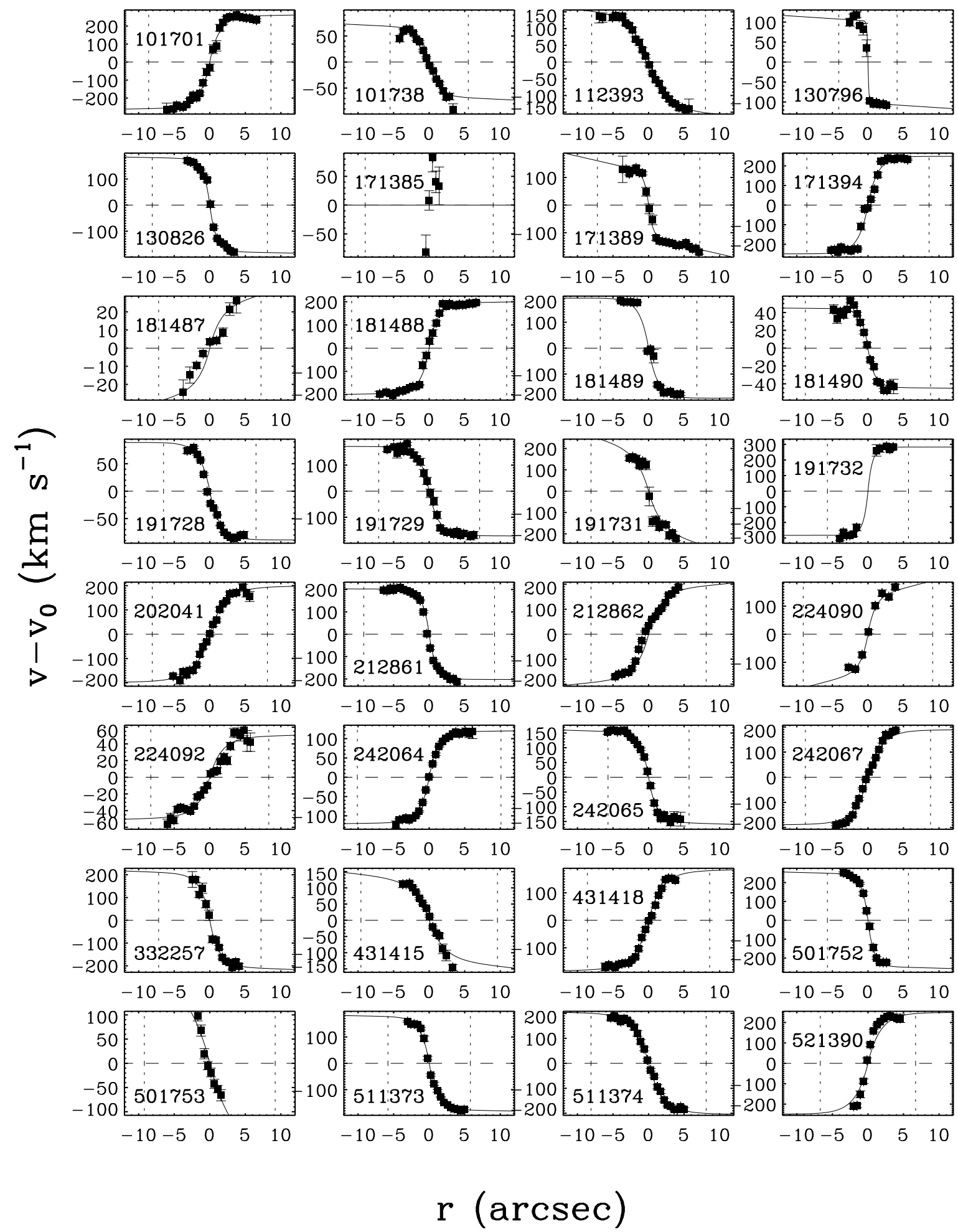

Fig. 1. The $\mathrm{H} \alpha$ rotation curves and their Polyex fits for the 32 spirals of the $z 0.2$ sample. The dotted lines show for each galaxy the position of $r_{83}$, the radius containing $83 \%$ of the light, and the solid line is the Polyex fit (when sufficient data was available).

the emission line, it was performed independently by two of us (AS, CM). The results were compared and a quality flag was put on the values. A width estimate was judged reliable when the two values agreed within the spectral resolution of the observations (i.e. $0.55 \AA$ ). The widths were transformed into rotation velocities using Eq. (2). This time, however, $F W H M_{i}$ is calculated using exposures of a FeAr hollow cathode calibration lamp, since there are very few bright sky lines over the relevant wavelength range. The calculated velocities $\left(\sigma_{\text {rot }}\right)$ are plotted against the rotation curve derived velocities for $\mathrm{H} \alpha$ on the bottom panel of Fig. 5 and given in the last column of Table 3. The rms of the residuals of the $\sigma_{\text {rot }}([\mathrm{OII}])-v_{\text {rot }}(\mathrm{H} \alpha)$ relation is $52.7 \mathrm{~km} \mathrm{~s}^{-1}$, which at any rotation width represents an error larger by a factor of $\sim 2.5$ than the relation for $\mathrm{H} \alpha$ alone. 


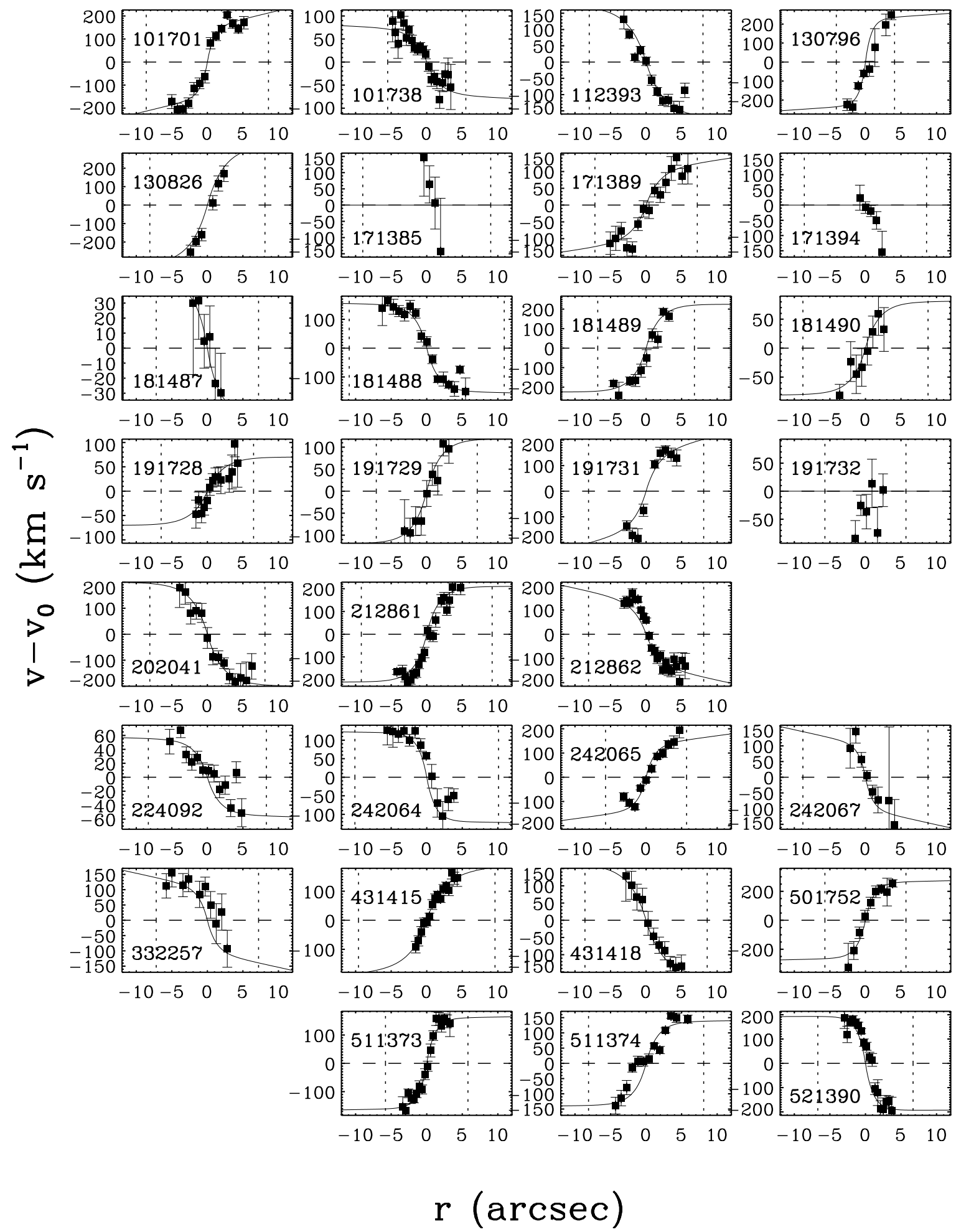

Fig. 2. The [OII] rotation curves and their Polyex fits for the galaxies which had detectable [OII] $\lambda 3727 \AA$ emission. The solid line represents the Polyex fit (when sufficient data was available), and the dotted lines show for each galaxy the position of $r_{83}$, the radius containing $83 \%$ of the light.

\subsection{Definition of the rotation classes}

When comes the time to perform the geometrical tests, the VVDS rotation velocities are used to separate the galaxies into two samples, one of slow and one of fast rotators. This is done in order to trace out the evolution of two different populations of galaxies by using the angular diameter test (see Papers I and III). These populations are defined as the "slow rotators", which have $0<v_{\text {rot }}<100 \mathrm{~km} \mathrm{~s}^{-1}$ and the "fast rotators" with $100<v_{\text {rot }}<200 \mathrm{~km} \mathrm{~s}^{-1}$. The analysis of the rotation curves and of the velocity histograms for the $z 0.2$ sample has shown that even considering the scatter in the relation between the different velocity indicators and the different spectral lines, similar criteria on rotation velocities can be applied across the different data sets to define the populations of objects that can act as standard rods or candles to perform the geometrical tests under the 

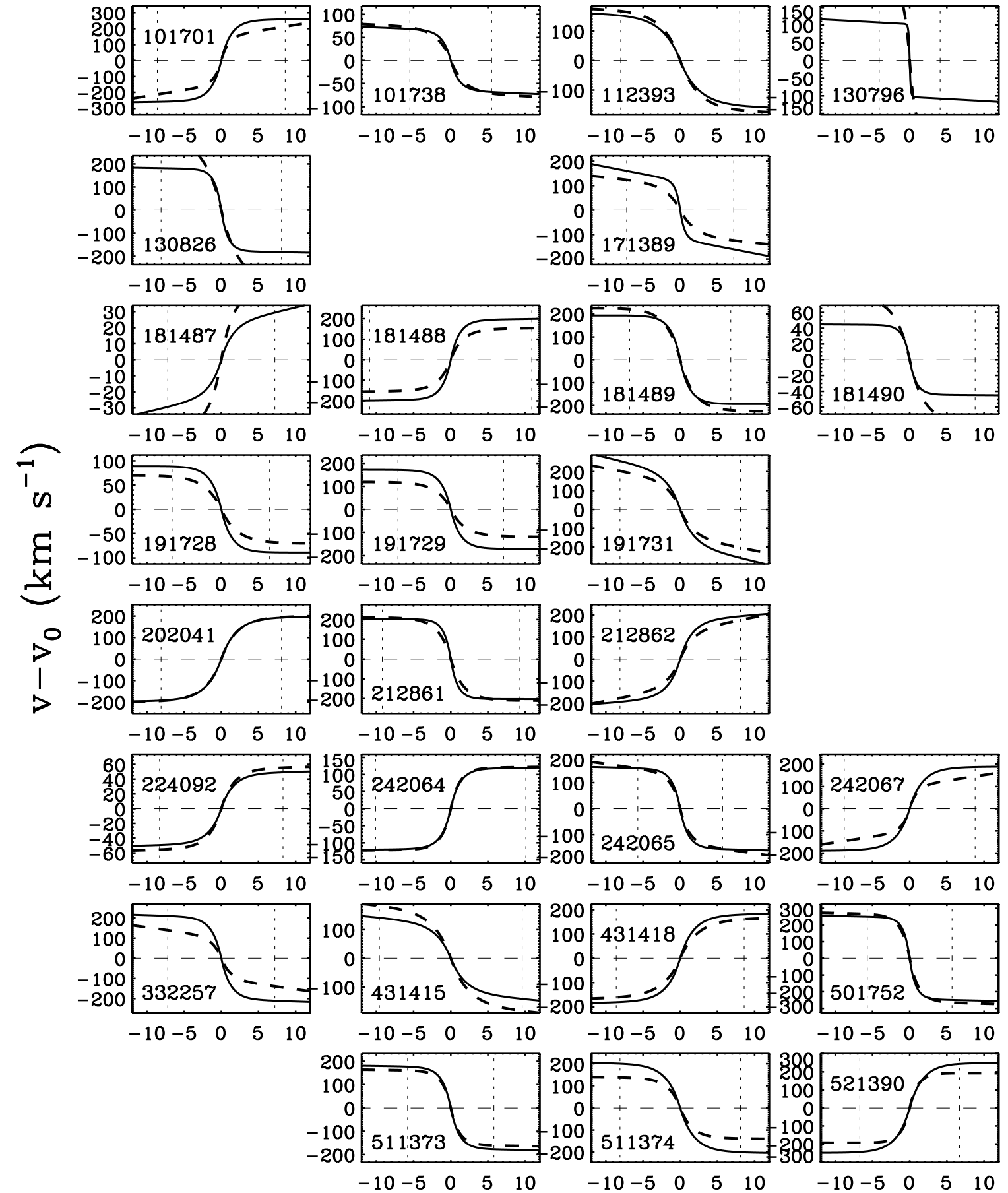

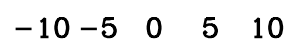
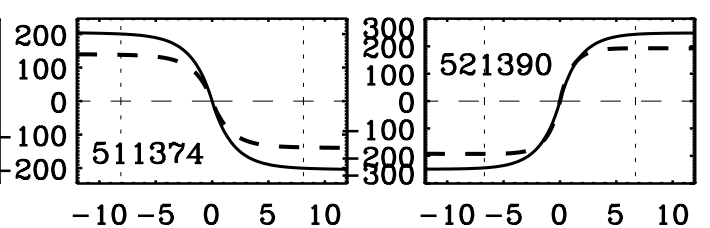

$\mathrm{r}(\operatorname{arcsec})$

Fig. 3. Polyex fits to the $\mathrm{H} \alpha$ (solid line) and to the [OII] (dashed line) rotation curves presented in Figs. 1 and 2, when both fits are available. Again, the vertical dotted lines show for each galaxy the position of $r_{83}$.

approach suggested in Paper I. Because of the restricted size of the $z 0.2$ sample and the very small number of "slow rotators" with reliable velocity measurements, these galaxies will not be used directly to perform the geometrical tests in Paper III. However, given the bridge that we have just established between low and high redshift velocity indicators, one could eventually use large data sets of nearby galaxies, such as SDSS, to complete the gap in redshift coverage between the very local SFI++ catalog (see next section), and the VVDS data.

\section{Calibrating the standard rods and standard candles}

In order to perform some geometrical tests such as the angular diameter test, not only will we need the well-defined samples of galaxies at $0.15 \lesssim z \lesssim 1.4$ but also reference values for the local Universe $(z \approx 0)$. Since the contributions of galaxy evolution and geometric signature are superposed, it is essential to determine the sizes and luminosities of the standard rods/candles for a local 
Table 2. Galactic rotation properties, from the $\mathrm{H} \alpha$ emission line.

\begin{tabular}{|c|c|c|c|c|c|}
\hline AGC \# & $\begin{array}{c}a_{1} \\
\mathrm{~km} \mathrm{~s}^{-1}\end{array}$ & $\begin{array}{c}a_{2} \\
\left(\operatorname{asec}^{-1}\right)\end{array}$ & $\begin{array}{c}a_{3} \\
\left(\operatorname{asec}^{-1}\right)\end{array}$ & $\begin{array}{c}v_{\text {rot }}\left(r_{83}\right) \\
\left(\mathrm{km} \mathrm{s}^{-1}\right)\end{array}$ & $\begin{array}{c}\sigma_{\text {rot }} \\
\left(\mathrm{km} \mathrm{s}^{-1}\right)\end{array}$ \\
\hline 101701 & 253.4 & 0.7800 & 0.00255 & $258.6 \pm 18.3$ & $275.6 \pm 12.5$ \\
\hline 101738 & 65.1 & 0.94 & 01000 & $68.3 \pm 10.8$ & $92.9 \pm 3.5$ \\
\hline 112393 & 151.6 & 0.4260 & 00436 & $151.8 \pm 6.7$ & $144.5 \pm 6.9$ \\
\hline 130796 & 102.9 & 9.2192 & 0.01120 & $107.6 \pm 17.5$ & $181.2 \pm 12.6$ \\
\hline 130826 & 177.4 & 1.1939 & 0.00319 & $182.0 \pm 10.0$ & $197.6 \pm 11.3$ \\
\hline 171385 & & & & & \\
\hline 171389 & 118.1 & 1.8150 & 0.05000 & $160.3 \pm 10.7$ & $179.4 \pm 20.4$ \\
\hline 171394 & 249.5 & 0.7820 & -0.00029 & $248.6 \pm$ & $236.8 \pm 25.4$ \\
\hline 181487 & 21.7 & 0.7317 & 0.05000 & $29.3 \pm 6.0$ & $62.0 \pm 3.5$ \\
\hline 181488 & 192.9 & 0.9066 & 0.00285 & $198.8 \pm 12.0$ & $200.6 \pm 22.9$ \\
\hline 181489 & 194.3 & 0.8190 & -0.00054 & $192.8 \pm 20.6$ & $175.9 \pm 8.7$ \\
\hline 181490 & 44.1 & 1.1869 & 0.00159 & $44.7 \pm 5.3$ & $78.7 \pm 3.4$ \\
\hline 191728 & 89.2 & 0.74 & -0.00040 & $88.2 \pm 7$ & $73.0=$ \\
\hline 191729 & 170.9 & & 0.00043 & 170. & $236.5 \pm 11.9$ \\
\hline 191731 & 183.6 & 0.6 & 0.05 & 256 & 17.2 \\
\hline 191732 & 282.4 & & & 4.1 & 50.4 \\
\hline 041 & 193.0 & & & 193. & $202.8=$ \\
\hline 212861 & 201.7 & & 0.00 & 202 & 199.1 \\
\hline 212862 & 173.8 & 0.6 & 0.01 & 193.8 & 144.3 \\
\hline 224090 & 131.0 & 1.0978 & 0.05000 & 190.7 & $203.7 \pm 10.7$ \\
\hline 224092 & 47.5 & 0.6303 & 0.00505 & $49.3 \pm$ & $77.9 \pm 5.9$ \\
\hline 242064 & 117.3 & 0.8463 & 0.00211 & $119.8 \pm$ & $131.6 \pm 7.0$ \\
\hline 242065 & 150.9 & 0.9871 & 0.00494 & 154.6 & $151.8 \pm 5.6$ \\
\hline 242067 & 186.1 & 0.5976 & 0.00119 & $187.0=$ & $131.4 \pm 5.6$ \\
\hline 332257 & 202.6 & 0.8316 & 0.00573 & $210.5 \pm$ & $192.6 \pm 18.2$ \\
\hline 431415 & 119.2 & & 0.02000 & 14.7 & $84.3 \pm 13.7$ \\
\hline 431418 & 176.3 & 0.5846 & 0.00356 & $180.5 \pm 10.8$ & $152.8 \pm 4.8$ \\
\hline 501752 & 243.9 & 1.3028 & 0.00417 & $249.5 \pm 6.2$ & $245.5 \pm 11.3$ \\
\hline 501753 & 186.1 & & 0.00399 & $182.0 \pm 12.3$ & $106.6 \pm 35.1$ \\
\hline 511373 & 175.2 & & & $177.2 \pm 8.1$ & $149.6 \pm 6.0$ \\
\hline & 199.5 & & & $200.3 \pm 7.9$ & $187.9 \pm 6.8$ \\
\hline 521390 & 248.8 & 0.5854 & 0.00000 & $243.9 \pm 30.8$ & $251.0 \pm 8.3$ \\
\hline
\end{tabular}

sample that is free from any evolution, and also the scatter in these relations (cf. Paper I, Paper III).

\subsection{A sample of galaxies in the local universe}

The SFI++ (S07), a sample of $\sim 4600$ local galaxies, is used for this purpose. They range in distance from 0 to $300 \mathrm{Mpc}$, with most objects within $100 \mathrm{Mpc}$. SFI++ builds on the earlier SFI (Spiral Field $I$-band) and SCI (Spiral Cluster $I$-band) samples published in a series of papers in the 1990s (e.g. Giovanelli et al. 1997; Haynes et al. 1999), but includes a significant amount of new data, plus reprocessing of the old values. Since SFI++ is a very large and homogeneous sample of nearby spiral galaxies, it is perfectly suited for the study of scaling relations in disc galaxies. The availability of Malmquist-corrected distances is of particular importance in determining physical sizes, making SFI++ the largest and best sample for this type of study, as evidenced by the low scatter in the $r-v_{\text {rot }}$ relation (see Sect. 4.2.2). An I-band Tully-Fisher template relation (Tully \& Fisher 1977) is presented in detail for the SCI subsample in Giovanelli et al. (1997), and for the larger SFI++ cluster sample in Masters et al. (2006, hereafter M06). Here, the values of half-light radius, absolute magnitude and surface brightness are used as calibration for high- $z$ data.

\subsection{Disc scaling relations}

Under the hierarchical scenario for the growth of structure, scaling relations between the luminosity, size and surface brightness and the rotation velocity of dark matter systems are predicted (Mo et al. 1998). The most commonly used of these, which is also the one with the least scatter, is the magnitude-rotation velocity (Tully-Fisher) relation.

\subsubsection{The magnitude-rotation velocity relation}

The Tully-Fisher relation for the SFI++ sample is shown in Fig. 6. The best fit to the Tully-Fisher relation at I-band has been derived by M06 for a late-type subset of these galaxies, for which accurate distances can be obtained through membership in nearby clusters of galaxies. We will from now on refer to this subset of galaxies as the "template sample". The Tully-Fisher relation from M06 is:

$M-5 \log h=-20.85 \pm 0.03-(7.85 \pm 0.1)\left(\log v_{\text {rot }}+\log 2-2.5\right)$.

We adopt this expression as our magnitude-velocity relation. M06 also give a precise estimate of the scatter around that relation. We recompute it here however for the complete SFI++ sample, to account for the broader morphological types included and the additional scatter coming from generally larger distance uncertainties. We measure the scatter to be $\sigma=0.56$ mag over the entire range of rotation velocities.

\subsubsection{The size-rotation velocity relation}

Since one of the main goals of this series of papers is to perform the angular diameter test on a sample of high redshift galaxies by using the relation between the physical size of a disc and its 
Table 3. Galactic rotation properties, from the $[\mathrm{OII}]$ emission line.

\begin{tabular}{cccccc}
\hline \hline AGC \# & $\begin{array}{c}a_{1} \\
\mathrm{~km} \mathrm{~s}^{-1}\end{array}$ & $\begin{array}{c}a_{2} \\
\left(\mathrm{asec}^{-1}\right)\end{array}$ & $\begin{array}{c}a_{3} \\
\left(\mathrm{asec}^{-1}\right)\end{array}$ & $\begin{array}{c}v_{\text {rot }}\left(r_{83}\right) \\
\left(\mathrm{km} \mathrm{s}^{-1}\right)\end{array}$ & $\begin{array}{c}\sigma_{\text {rot }} \\
\left(\mathrm{km} \mathrm{s}^{-1}\right)\end{array}$ \\
\hline 101701 & 148.9 & 1.1659 & 0.05000 & $212.8 \pm 23.5$ & $217.0 \pm 53.3$ \\
101738 & 70.5 & 0.5846 & 0.01000 & $71.5 \pm 18.4$ & $84.7 \pm 13.2$ \\
112393 & 166.7 & 0.4304 & 0.00436 & $167.1 \pm 9.1$ & $186.4 \pm 56.6$ \\
130796 & 226.2 & 1.2712 & 0.01120 & $235.4 \pm 45.9$ & $260.1 \pm 47.1$ \\
130826 & 318.4 & 0.4384 & 0.00319 & $317.0 \pm 13.5$ & $185.2 \pm 111.4$ \\
171385 & $\ldots$ & $\ldots$ & $\ldots$ & $\ldots$ & $\ldots$ \\
171389 & 103.3 & 0.5794 & 0.03000 & $123.5 \pm 24.2$ & $205.8 \pm 73.7$ \\
171394 & $\ldots$ & $\ldots$ & $\ldots$ & $\ldots$ & $283.8 \pm 86.2$ \\
181487 & 42.7 & 0.6560 & 0.00288 & $43.2 \pm 5.8$ & $121.5 \pm 41.7$ \\
181488 & 150.0 & 0.6544 & 0.00285 & $154.5 \pm 15.2$ & $212.4 \pm 52.2$ \\
181489 & 226.5 & 0.5540 & -0.00054 & $220.3 \pm 35.9$ & $296.2 \pm 83.2$ \\
181490 & 79.5 & 0.5449 & 0.00159 & $79.9 \pm 11.7$ & $116.6 \pm 54.6$ \\
191728 & 70.5 & 0.4750 & -0.00040 & $67.1 \pm 16.5$ & $176.6 \pm 31.2$ \\
191729 & 118.7 & 0.5453 & 0.00043 & $116.6 \pm 18.6$ & $271.8 \pm 78.3$ \\
191731 & 145.2 & 0.6494 & 0.05000 & $202.7 \pm 43.6$ & $194.6 \pm 59.1$ \\
191732 & $\ldots$ & $\ldots$ & $\ldots$ & $\ldots$ & $279.1 \pm 72.9$ \\
202041 & 195.2 & 0.4955 & 0.00204 & $194.8 \pm 14.0$ & $225.3 \pm 60.8$ \\
212861 & 209.5 & 0.6183 & 0.00037 & $209.5 \pm 24.4$ & $129.9 \pm 37.8$ \\
212862 & 126.6 & 0.6494 & 0.05000 & $176.8 \pm 31.1$ & $155.4 \pm 34.5$ \\
224090 & $\ldots$ & $\ldots$ & $\ldots$ & $\ldots$ & $\ldots$ \\
224092 & 53.5 & 0.6303 & 0.00505 & $55.5 \pm 13.6$ & $117.6 \pm 34.1$ \\
242064 & 119.2 & 0.8766 & 0.00211 & $121.7 \pm 15.7$ & $148.2 \pm 44.7$ \\
242065 & 131.8 & 0.9146 & 0.03000 & $153.6 \pm 28.1$ & $109.1 \pm 20.9$ \\
242067 & 100.9 & 1.1304 & 0.05000 & $145.1 \pm 29.0$ & $166.3 \pm 76.6$ \\
332257 & 102.5 & 0.9018 & 0.05000 & $139.4 \pm 19.1$ & $141.5 \pm 66.0$ \\
431415 & 161.7 & 0.4619 & 0.01500 & $182.9 \pm 6.6$ & $92.6 \pm 29.5$ \\
431418 & 159.4 & 0.4397 & 0.00356 & $160.5 \pm 9.0$ & $229.1 \pm 43.2$ \\
501752 & 260.5 & 0.8905 & 0.00417 & $265.0 \pm 40.8$ & $369.2 \pm 145.5$ \\
501753 & 137.1 & 0.7214 & 0.00399 & $142.0 \pm 5.6$ & $175.7 \pm 49.0$ \\
511373 & 158.9 & 0.9331 & 0.00283 & $160.8 \pm 13.7$ & $189.7 \pm 30.7$ \\
511374 & 136.8 & 0.6494 & 0.00185 & $138.1 \pm 29.6$ & $182.7 \pm 28.7$ \\
521390 & 194.1 & 0.9440 & -0.00068 & $192.9 \pm 11.7$ & $307.2 \pm 53.1$ \\
\hline & & & & &
\end{tabular}

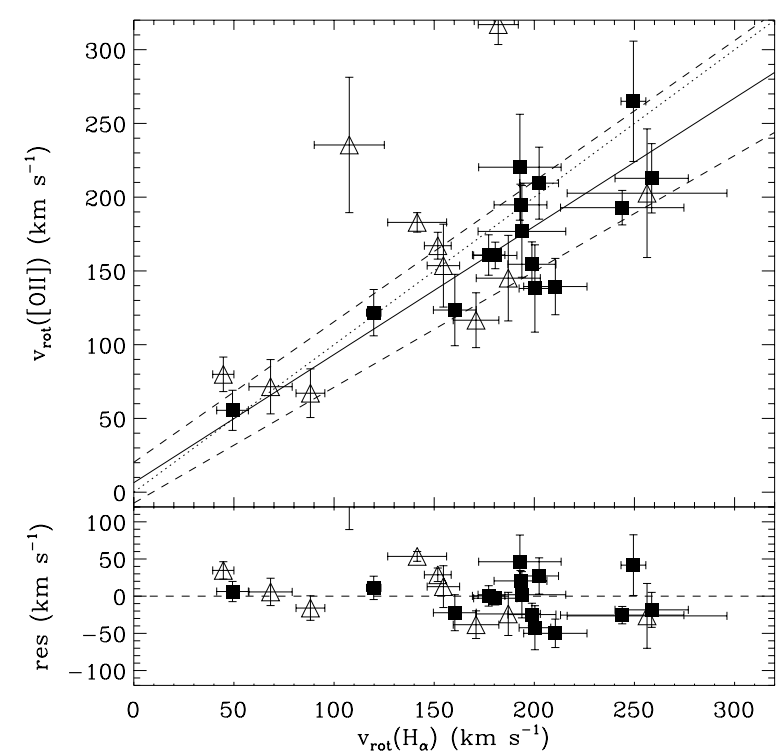

Fig. 4. Comparison of the rotational velocities derived from the rotation curves for $\mathrm{H} \alpha$ and [OII]. Top panel: the solid line is the best fit obtained by an ordinary least-squares regression, excluding the two outlying points. The dashed lines are the maximum and minimum slopes determined by the errors on the parameters of the best-fit linear relation and the dotted line corresponds to a 1:1 relation. Bottom panel: the residuals of the best-fit model. In both panels, the filled symbols represent the 15 galaxies with the most secure rotation velocity estimates, while the open symbols are those galaxies with poorer rotation curve extent, either in $\mathrm{H} \alpha$ or [OII]. rotation velocity to select standard rods, it is most important to derive a reliable expression for the $z=0$ size-rotation velocity $\left(r-v_{\text {rot }}\right)$ relation. S07 published two sets of sizes for the galaxies in the SFI++ sample: $r_{83}$, the radius containing $83 \%$ of the light, and $r_{23.5}^{0}$ the radius corresponding to the $\mu=23.5$ isophote, corrected to face-on (Giovanelli et al. 1995, hereafter G95). While values of the disc scale length $r_{\mathrm{d}}$ are available for SFI++ galaxies, they tend to be unreliable and produce scaling relations with large scatter (Giovanelli et al. 1994). In Figs. 7 and 8 we present the $r-v_{\text {rot }}$ for $r_{83}$ and $r_{23,5}^{\mathrm{o}}$, respectively. The scatter around the best-fit bivariate linear fit is smallest when $r_{235}^{\mathrm{O}}$ is used. The Pearson correlation coefficient of the relation is 0.92 for $r_{23.5}^{\mathrm{o}}$ and 0.67 for $r_{83}$. We will therefore not consider $r_{83}$ any further, but the parameters of the best direct, inverse and bivariate linear fits to the $r_{83}-v_{\text {rot }}$ relation are given in Table 4 , as well as those for $r_{235}^{\mathrm{o}}-v_{\text {rot }}$. The top half of the table presents the fit results for the 699 galaxies of the template sample, and the lower half the parameters for all galaxies in SFI++ with size estimates, a total of 4596 galaxies.

The residuals of the best fit to the $r_{23.5}^{0}-v_{\text {rot }}$ relation are presented in the upper panel of Fig. 9. The bottom panel of the same figure shows the average scatter in the residuals as a function of rotation velocity, in bins containing an equal number of galaxies. The scatter is larger for the smallest galaxies, but averages 0.069 for galaxies with $v_{\text {rot }}>100 \mathrm{~km} \mathrm{~s}^{-1}$. The average scatter for the entire sample is 0.073 in units of $\log \left(\mathrm{kpc} \mathrm{h}^{-1}\right)$.

The smaller scatter in the $r_{23.5}^{\mathrm{o}}-v_{\text {rot }}$ relation is explained by the fact that $r_{23.5}$ as opposed to $r_{83}$ is measured where the disc is close to optically thin, so it is possible to correct it for 


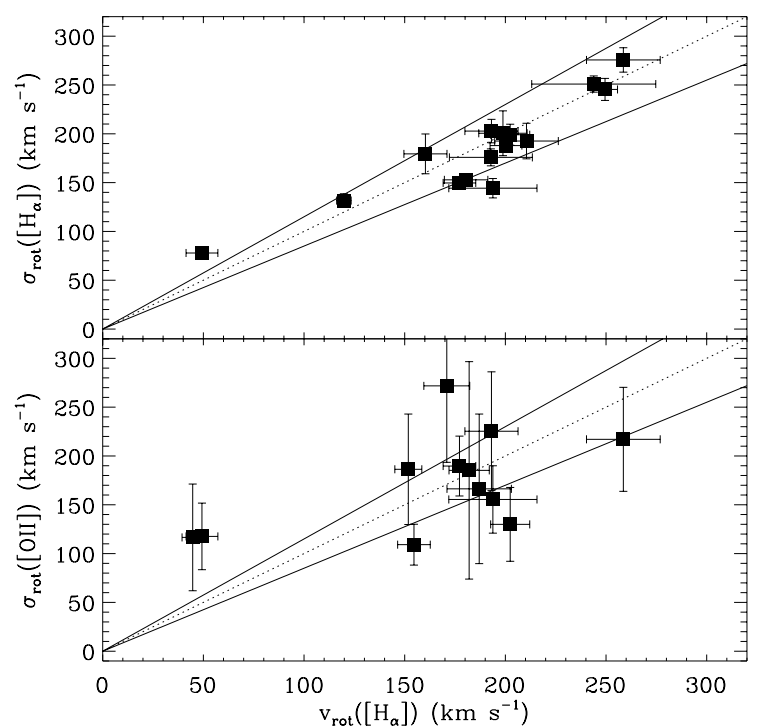

Fig. 5. Comparison of the rotational velocities derived from the rotation curves $\left(v_{\text {rot }}\right)$ and from the one-dimensional collapsed spectra $\left(\sigma_{\text {rot }}\right)$ for $\mathrm{H} \alpha$ (top panel) and [OII] (bottom panel). In each pannel the dotted line corresponds to a 1:1 relation, and the continuous lines a deviation of $15 \%$ from it.

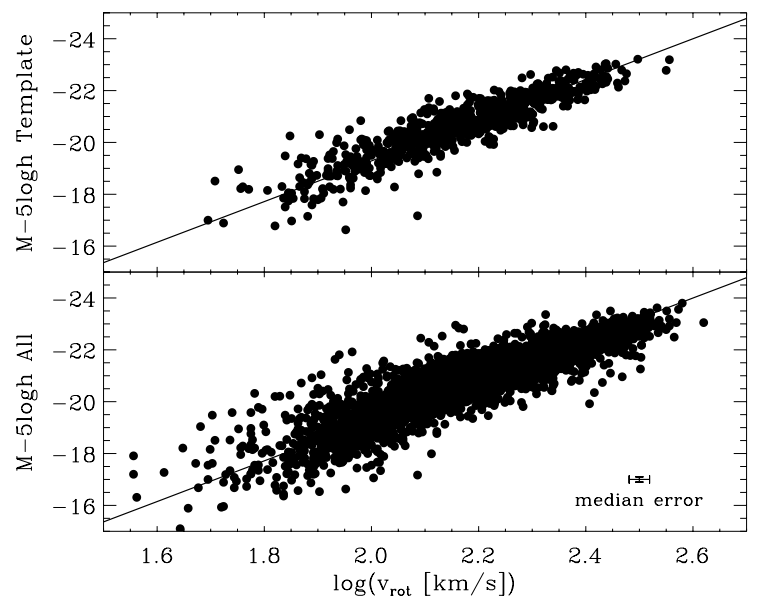

Fig. 6. Magnitude-rotation velocity relation for the SFI++ galaxies. Absolute magnitude at $I$-band, corrected for extinction and corrected to face-on (S07), plotted against rotation velocity. The solid line in each panel is the template Tully-Fisher relation derived by M06, using only the subset of galaxies with the most accurate distances (top panel). The bottom panel includes all the SFI++ galaxies.

inclination effects. While no inclination correction has been attempted for $r_{83}$, the values of $r_{23.5}$ plotted in Fig. 8 have been corrected for inclination to their face-on values using Eq. (7) of G95, where a complete analysis of the inclination dependence of isophotal radii was done. We have further corrected these radii for foreground galactic extinction as:

$r_{23.5}^{\mathrm{cor}}=r_{23.5}^{\mathrm{o}}+\frac{A_{i} r_{\mathrm{d}}}{1.086}$,

where $r_{23.5}^{\mathrm{o}}$ is the observed value corrected to face-on, $A_{i}$ the galactic extinction at $I$-band derived from the DIRBE dust maps (Schlegel et al. 1998), and $r_{\mathrm{d}}$ the disc scale length ${ }^{2}$. The small

${ }^{2}$ Estimates of $r_{\mathrm{d}}$ are available for SFI++ galaxies, and while they are less reliable size measures than $r_{83}$ or $r_{23.5}$, they are useful for applying small corrections to the latter.

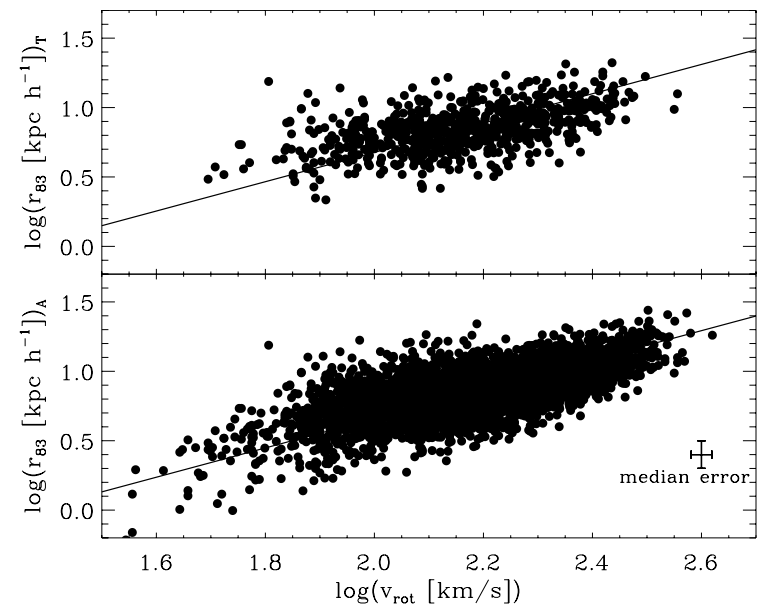

Fig. 7. $r-v_{\text {rot }}$ relation for the template sample (top panel) and for the full SFI++ sample (bottom panel). The quantity plotted is $r_{83}$, the radius encompassing $83 \%$ of the light. No attempt is made to correct $r_{83}$ for inclination. In each case the solid line is the best bisector linear fit to the relation, the parameters of which are given in Table 4.

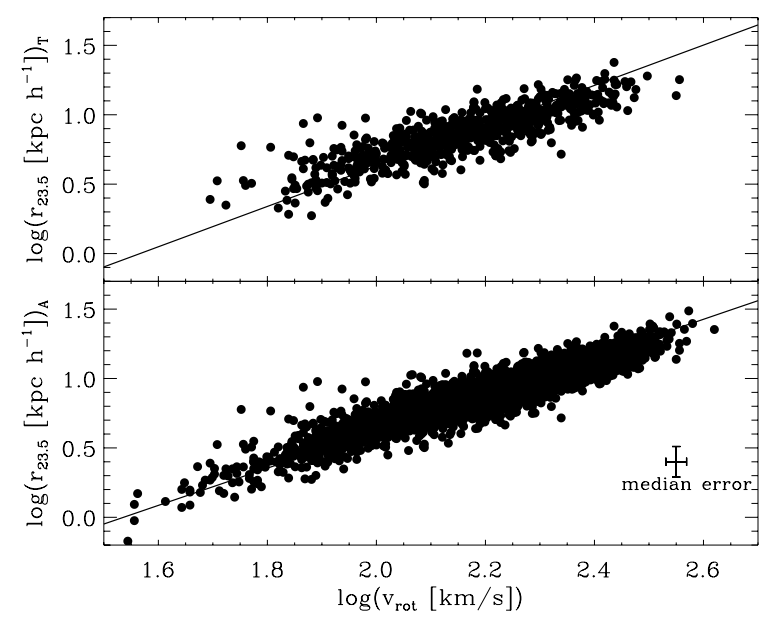

Fig. 8. $r-v_{\text {rot }}$ relation for the template sample (top panel) and for the full SFI++ sample (bottom panel). The quantity plotted is $r_{23.5}$, the radius corresponding to the $\mu=23.5$ isophote, after corrections for inclination, foreground extinction and cosmological stretch. In each case the solid line is the best bisector linear fit to the relation, the parameters of which are given in Table 4 .

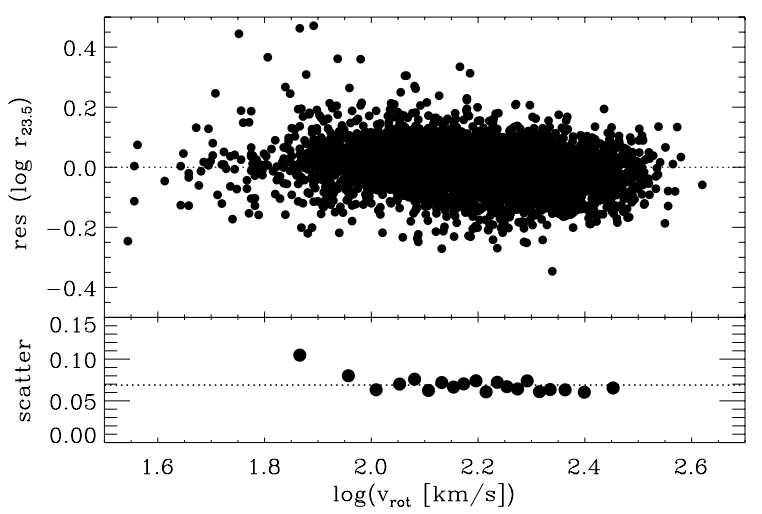

Fig. 9. Top panel: residuals of the $r_{235}^{\mathrm{o}}-v_{\text {rot }}$ relation for the full SFI++ sample. Bottom panel: average scatter in the residuals as a function of rotation velocity. The galaxies were divided in bins containing an equal number of objects. The average scatter for the entire sample is 0.073 . The dotted line is at 0.068 , which is the average value for galaxies with $v_{\text {rot }}>100 \mathrm{~km} \mathrm{~s}^{-1}$, where the scatter reaches a plateau. 
scatter in the $r_{23.5}^{\mathrm{o}}-v_{\text {rot }}$ can also be partly explained by the care that has been taken in deriving distances for the SFI++ galaxies. These distances were calculated by taking into account the peculiar velocity of each galaxy. For field galaxies, the peculiar velocities are derived from the systemic velocity of the galaxies and the absolute magnitude compared to the values predicted by the Tully-Fisher relation. For galaxies in groups, the peculiar velocity is the weighted average of the peculiar velocities of the group members (S07). The distances are further corrected for the Malmquist bias.

Since the slopes and zero-points of the relations can depend on the fitting technique, we briefly comment on the method used here. All linear fits were done in IDL using the task LINMIX_ERR, available from the IDL Astronomy User's Library, which incorporates a Bayesian approach to linear regressions taking into account measurement errors on both $X$ and $Y$ variables. This marks another improvement over previous determinations of the $r-v_{\text {rot }}$ relation: error measurements on the values of $r_{23.5}$ are calculated with precision and included in the fits. The error values we use include a $5 \%$ measurement error on $r_{23.5}$, the uncertainty on the coefficient $\beta$ of the expression used to correct radii to face-on values (G95), the uncertainty on the ellipticity of the galaxies used in the inclination correction, and the distance uncertainty. This last term dominates the total error on $r_{23,5}^{\mathrm{o}}$.

Direct comparisons between the scaling relations and scatter derived for different galaxy samples are complicated by the selection functions and variable definitions adopted in different studies. For these reasons, a quantitative comparison between our results and that of other groups is beyond the scope of this work. Here, we simply emphasize the unprecedentedly small scatter in the $r_{23.5}-v_{\text {rot }}$ relation, which is a factor of a few smaller than all previous estimates (e.g. Courteau et al. 2007). A careful analysis of this scatter and its implications for disk galaxy formation models will be explored in a forthcoming study.

To calibrate the geometrical tests performed in Paper III, the relation between the half-light radius $r_{50}$ and rotation velocity needs to be derived. As stated in Paper I, it is crucial for these tests to use a metric rather than an isophotal definition for the size of the standard rods. While it would be straightforward to calculate $r_{50}$ from $r_{83}$ (for an exponential disc $r_{50}=0.52 r_{83}$ ), we rather choose to calculate it from $r_{23.5}$ since the $r_{23.5}-v_{\text {rot }}$ relation is significantly tighter. Assuming that the discs obey an exponential profile, the half-light radius is simply $r_{50}=1.68 r_{\mathrm{d}}$. Instead of using relatively inaccurate individual estimates of $r_{\mathrm{d}}$ to generate $r_{50}$ for each galaxy, we generate a proxy for this value using $r_{23.5}$. Specifically, we plot $r_{\mathrm{d}} / r_{23.5}$ as a function of $M_{I}$ for the SFI++, and use a linear fit to this relation to obtain $r_{\mathrm{d}} / r_{23.5}$ for each galaxy given its magnitude. An estimate of $r_{\mathrm{d}}^{\mathrm{o}}$ for each system is then obtained by multiplying this value by $r_{23.5}$ and correcting to face-on with Eq. (5) of G95. The values of $r_{50}$ that we adopt are derived from these corrected scale-lengths. The $r_{50}-v_{\text {rot }}$ relation is presented in Fig. 10, and the parameters of the best-fit linear relations are in Table 4 . The scatter in the $r_{50}-v_{\text {rot }}$ relation when $r_{50}$ is derived from $r_{23.5}$ is considerably smaller than if derived from $r_{83}$ (see Table 4 ) or even more so from the measured values of $r_{\mathrm{d}}$. The $r_{50}-v_{\text {rot }}$ relation is central to the geometrical tests of cosmological models performed in Paper III.

\subsubsection{The surface brightness-rotation velocity relation}

The surface brightness-rotation velocity $\left(\mu-v_{\text {rot }}\right)$ relation is the least often used of these three scaling relations, as it intrinsically has more scatter than both the Tully-Fisher and the $r-v_{\text {rot }}$

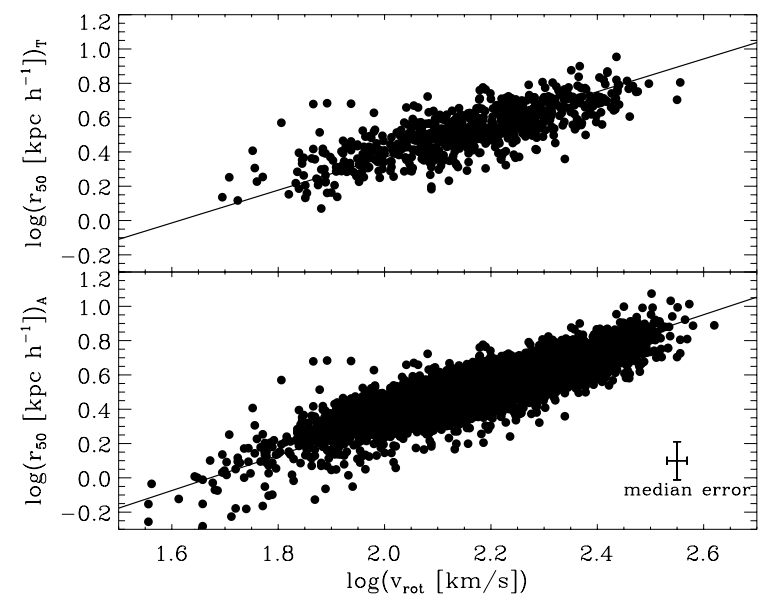

Fig. 10. $r-v_{\text {rot }}$ relation for the template sample (top panel) and for the full SFI++ sample (bottom panel). The quantity plotted is $r_{50}$, which has been computed as $r_{50}=1.68 r_{\mathrm{d}}$, where $r_{\mathrm{d}}$ the disc scale length was estimated by scaling the value of $r_{23.5}$ by a factor depending on the magnitude of the galaxies. In each case the solid line is the best bisector linear fit to the relation, the parameters of which are given in Table 4.

relations. This larger scatter is due at least in part to a stronger dependency of the $\mu-v_{\text {rot }}$ relation on the spin parameter of the disc $\lambda$; while the $M-v_{\text {rot }}$ relation is independent of $\lambda$, this parameter appears linearly in the $r-v_{\text {rot }}$ relation and quadratically in the $\mu-v_{\text {rot }}$ relation (Mo et al. 1998).

In Fig. 11 we show the $\mu-v_{\text {rot }}$ relation both for the template sample and for the entire SFI++ relation. The quantity plotted is the average surface brightness within $r_{50}$, calculated as:

$\mu_{50}=m_{\mathrm{tot}}+5 \log \left(r_{50}\right)+2.5 \log (2 \pi)$.

The best fit relation to the entire SFI++ sample, using an ordinary least squares bisector method, is:

$\mu_{50}=(29.67 \pm 0.22)-(4.43 \pm 0.09) \log \left(v_{\text {rot }}\right)$.

The $1 \sigma$ scatter in the residuals of this best fit relation is $0.78 \mathrm{mag} \mathrm{arcsec}^{-2}$.

\section{Conclusions}

The main goal of this paper was to provide calibration in two different ways for the angular diameter test that we performed on data from the VVDS: (1) by directly comparing velocity indicators commonly used in the local and distant Universe, and (2) by using a large sample of nearby galaxies to provide zero points for the radius-rotation velocity, magnitude-rotation velocity and surface brightness-rotation velocity relations. Previous studies have focused on comparing the $\mathrm{H} \alpha$ or $[\mathrm{OII}]$ rotation velocities with $\mathrm{HI}$ data for nearby galaxies. Kobulnicky \& Gebhardt (2000) have used a sample of 22 nearby galaxies to compare [OII] and HI linewidths. Their galaxy sample was smaller and less homogeneous that the one used in this study. They found the two sets of velocity estimates to be consistent within $10 \%$ overall, with the [OII] underestimating the $\mathrm{HI}$ rotation velocity by up to $50 \%$ in a few extreme cases. In the local Universe, the consistency of the $\mathrm{HI}$ and $\mathrm{H} \alpha$ rotation velocities was also established (see for example Courteau 1997; Vogt et al. 2004). Most recently, Catinella et al. (2007) has shown that on average HI widths are larger than $\mathrm{H} \alpha$ ones by $\sim 10 \%$ for a galaxy with $w=100 \mathrm{~km} \mathrm{~s}^{-1}$ and that optical rotational widths measured from velocity histograms are affected by systematic biases and therefore are less reliable that 
Table 4. Coefficients of best direct, inverse and bivariate linear fits to the $r-v_{\text {rot }}$ relation, $\log \left(r\left[\mathrm{kpc} \mathrm{h}^{-1}\right]\right)=a+b \log \left(v_{\mathrm{rot}}\left[\mathrm{km} \mathrm{s}^{-1}\right]\right)$.

\begin{tabular}{|c|c|c|c|c|c|c|c|}
\hline Relation & $a_{\mathrm{dir}}$ & $b_{\text {dir }}$ & $a_{\text {inv }}$ & $b_{\text {inv }}$ & $a_{\mathrm{bi}}$ & $b_{\mathrm{bi}}$ & $\sigma_{\mathrm{bi}}\left(\log \left(r\left[\mathrm{kpc} \mathrm{h}^{-1}\right]\right)\right)$ \\
\hline Template galaxies & & & & & & & \\
\hline$r_{83}-v_{\mathrm{rot}}$ & $-0.36 \pm 0.08$ & $0.56 \pm 0.04$ & $-3.56 \pm 0.29$ & $2.04 \pm 0.13$ & $-1.43 \pm 0.06$ & $1.06 \pm 0.03$ & 0.16 \\
\hline$r_{23.5}-v_{\text {rot }}$ & $-1.35 \pm 0.06$ & $1.03 \pm 0.03$ & $-2.27 \pm 0.07$ & $1.45 \pm 0.03$ & $-1.77 \pm 0.06$ & $1.22 \pm 0.03$ & 0.10 \\
\hline$r_{50}-v_{\text {rot }}$ & $-1.06 \pm 0.05$ & $0.73 \pm 0.02$ & $-2.16 \pm 0.09$ & $1.24 \pm 0.04$ & $-1.54 \pm 0.04$ & $0.96 \pm 0.02$ & 0.11 \\
\hline All SFI++ & & & & & & & \\
\hline$r_{83}-v_{\text {rot }}$ & $-0.80 \pm 0.03$ & $0.76 \pm 0.02$ & $-2.85 \pm 0.07$ & $1.69 \pm 0.03$ & $-1.45 \pm 0.07$ & $1.06 \pm 0.03$ & 0.14 \\
\hline$r_{23.5}-v_{\text {rot }}$ & $-1.64 \pm 0.02$ & $1.15 \pm 0.01$ & $-2.06 \pm 0.03$ & $1.34 \pm 0.01$ & $-1.84 \pm 0.02$ & $1.24 \pm 0.01$ & 0.07 \\
\hline$r_{50}-v_{\mathrm{rot}}$ & $-1.39 \pm 0.02$ & $0.88 \pm 0.01$ & $-2.09 \pm 0.04$ & $1.20 \pm 0.02$ & $-1.71 \pm 0.02$ & $1.02 \pm 0.01$ & 0.08 \\
\hline
\end{tabular}

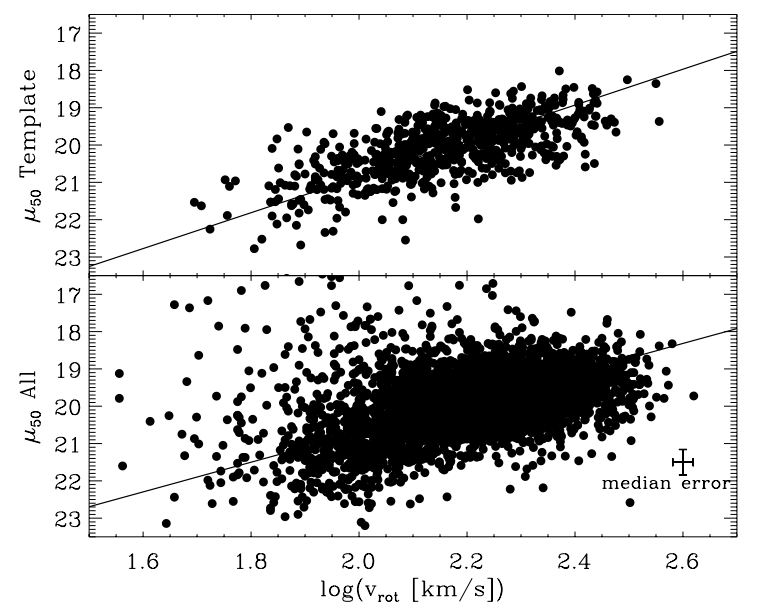

Fig. 11. Surface brightness-rotation velocity relation for the template sample (top panel) and for the complete SFI++ sample (bottom panel). Surface brightness at $I$-band within $r_{50}$, the radius containing $50 \%$ of the light, plotted against rotation velocity. The top panel uses only the smaller sample used by M06 to derive the template Tully-Fisher relation, while the bottom panel includes all the SFI++ galaxies.

those derived by using the full spatial information through the fitting of the rotation curves.

For this study, we first analysed the rotation curves for all the galaxies in our sample and concluded that the rotation velocities derived from the [OII] $\lambda 3727 \AA$ line translate directly into the velocities obtained from $\mathrm{H} \alpha$ rotation curves, given that the sampling of the [OII] rotation curve extends far enough to reach the region of constant (or slowly increasing) velocity. Even under this condition and as shown in Fig. 4, there is scatter in the relation of about $30 \mathrm{~km} \mathrm{~s}^{-1}$. The next step was to compare the techniques used to determine rotation velocities in the local Universe ( $\mathrm{H} \alpha$ rotation curves) and at high redshift ([OII] velocity histograms). Once again, there is a direct relation between the two but significant scatter on the order of $50 \mathrm{~km} \mathrm{~s}^{-1}$ (see Fig. 5).

We conclude that all the rotation velocity indicators studied here give comparable results, but before combining samples analysed with different methods one should be aware of the important scatter in the different relations. This scatter is due mostly to the poorer spatial extent of the [OII] rotation profiles compared to the $\mathrm{H} \alpha$ ones obtained with the same integration time, and can be as large as $25 \%$ for a galaxy with $w=200 \mathrm{~km} \mathrm{~s}^{-1}$ or $100 \%$ if $w=50 \mathrm{~km} \mathrm{~s}^{-1}$ in the case of the $\sigma_{\text {rot }}([\mathrm{OII}])-v_{\text {rot }}(\mathrm{H} \alpha)$ relation (Fig. 5). Even considering this large scatter, the fact that there is no systematic deviation from a $1: 1$ relation allows us to use both methods in their respective context to divide galaxies in classes based on rotation velocity, as required to perform the geometrical tests with the high redshift sample (see Paper III). In Paper I it was showed that this is a viable way of selecting standard rods for the purpose of the angular diameter test. Since one cannot perform the test only with high redshift data, it was of great importance to establish that a set of local calibrators can be reliably compared to it, even if the rotation measure is different.

Once it is established that the various rotation indicators can be reliably compared, the next critical step is to derive the scaling relations between luminosity, size, surface brightness and rotation velocity for a set of nearby galaxies, free from any evolution. We used the SFI++ catalog, a very large homogeneous sample of nearby spiral galaxies with $I$-band photometry and rotation information obtained either through optical long-slit spectroscopy or HI measurements. We have particularly focussed our attention on the $r-v_{\text {rot }}$ relation which is central to the cosmological tests performed in the other papers of this series. We have shown that by carefully correcting sizes for inclination and extinction effects as well as obtaining accurate distances to the galaxies, the $r_{23.5}-v_{\text {rot }}$ relation can be almost as tight as the Tully-Fisher relation, as judged by their Pearson correlation coefficients. The relation derived using $r_{23.5}$ as the size measurement is considerably tighter than the ones obtained using the disc scale length $r_{\mathrm{d}}$ or a radius defined to contain a given fraction of the light of the galaxy (in our case $r_{83}$ or $r_{50}$ ). The next phase for this study on scaling relations will be to see what constraints our $r-v_{\text {rot }}$ relation imposes on models for disc galaxy formation, for example the range of spin parameters its tight scatter allows. These scaling relations also offer exciting possibilities for the study of disc galaxy evolution. Since they provide accurate values for luminosity, size and surface brightness as a function of rotation velocity, measurements of the properties for galaxies with the same rotation velocity (i.e. hosted in a dark halo of the same mass) over a large redshift range can provide in a new and unique way information about the evolution of galaxies and a mean of testing predictions from models under the hierarchical scenario for the growth of structures. A pilot study is presented in the next paper of this series.

Acknowledgements. This work has been partially supported by NSF grants AST-0307661 and AST-0307396 and was done while AS was receiving a fellowship from the Fonds de recherche sur la Nature et les Technologies du Québec. KLM is supported by the NSF grant AST-0406906. K.S. is a Jansky Fellow of NRAO. This research has made use of the NASA/IPAC Extragalactic Database (NED) which is operated by the Jet Propulsion Laboratory, California Institute of Technology, under contract with the National Aeronautics and Space Administration.

\section{References}

Catinella, B., Haynes, M. P., \& Giovanelli, R. 2005, AJ, 130, 1037 Catinella, B., Haynes, M. P., \& Giovanelli, R. 2007, AJ, 134, 334 Cooray, A. R. 1998, A\&A, 333, L71

Cooray, A., Hu, W., Huterer, D., \& Joffre, M. 2001, ApJ, 557, 7 
Courteau, S. 1997, AJ, 114, 2402

Courteau, S., Dutton, A. A., van den Bosch, F., et al. 2007, [arXiv : 0708. 0422]

Dalcanton, J. J., Spergel, D. N., \& Summers, F. J. 1997, ApJ, 482, 659

Davis, M., Newman, J., Faber, S., \& Phillips, A. 2001, in Deep Fields, Proceedings of the ESO/ECF/STScI Workshop, ed. S. Christiani, A. Renzini, \& R. E. Williams (Garching: Springer), 241

Dutton, A. A., van den Bosch, F. C., Dekel, A., \& Courteau, S. 2007, ApJ, 654, 27

Förster Schreiber, N. M., Genzel, R., Lehnert, M. D., et al. 2006, ApJ, 645, 1062 Giovanelli, R., \& Haynes, M. P. 2002, ApJ, 571, L107

Giovanelli, R., Haynes, M. P., Salzer, J. J., et al. 1994, AJ, 107, 6

Giovanelli, R., Haynes, M. P., Salzer, J. J., et al. 1995, AJ, 110, 1059 (G95)

Giovanelli, R., Haynes, M.P., Herter, T., et al. 1997, AJ, 113, 53

Haynes, M. P., Giovanelli, R., Salzer, J. J., et al. 1999, AJ, 117, 1668

Isobe, T., Feigelson, E. D., Akritas, M. G., \& Babu, G. J. 1990, ApJ, 364, 104

Kobulnicky, H.A., \& Gebhardt, K. 2000, AJ, 119, 1608

Le Fèvre, O,. et al. 2001, in Deep Fields, Proceedings of the ESO/ECF/STScI

Workshop, ed. S. Christiani, A. Renzini, \& R.E. Williams (Garching: Springer), 236

Marinoni, C., Saintonge, A., Giovanelli, R., et al. 2008, A\&A, 478, 43

Marinoni, C., Saintonge, A., Contini, T. et al. 2008, A\&A, 478, 71
Masters, K. L., Springob, C. M., Haynes, M. P., \& Giovanelli, R. 2006, ApJ, 653, 861 (M06)

Mo, H. J., Mao, S., \& White, S. D. M. 1998, MNRAS, 295, 319

Oke, J. B., \& Gunn, J. E. 1982, PASP, 94, 586

Persic, M., Salucci, P., \& Stel, F. 1996, MNRAS, 281, 27

Sandage, A. 1972, ApJ, 173, 485

Schlegel, D. J., Finkbeiner, D. P., \& Davis, M. 1998, ApJ, 500, 525

Sereno, M. 2003, A\&A, 412, 341

Somerville, R. S., Barden, M., Rix, H.-W., et al. 2006,

[arXiv: astro-ph/0612428]

Springob, C. M., Masters, K. L., Haynes, M. P., Giovanelli, R., \& Marinoni, C. 2007, ApJS, 172, 599 (S07)

Stoughton, C., Lupton, R. H., Bernardi, M., et al. 2002, AJ, 123, 485

Swaters, R. A., Madore, B. F., \& Trewhella, M. 2000, ApJ, 531, L107

Tody, D. 1993, Astronomical Data Analysis Software and Systems II, ASP Conf. Ser. 52, 173

Tully, R. B., \& Fisher, J. R. 1977, A\&A, 54, 661

van den Bosch, F. C., Robertson, B. E., Dalcanton, J. J., \& de Blok, W. J. G. 2000, AJ, 119, 1579

Vogt, N. P., Haynes, M. P., Herter, T., \& Giovanelli, R. 2004, ApJ, 127, 3273

Weiner, B. J., Willmer, C. N. A., Faber, S. M., et al. 2006, ApJ, 653, 1027 